\title{
EDITORIAL
}

\section{The First Engineering Journal from Nature Publishing Group: Microsystems \& Nanoengineering}

\author{
Microsystems \& Nanoengineering (2015) 1, 15003; doi:10.1038/ \\ micronano.2015.3; Published online: 28 May 2015
}

After extensive collaboration and detailed planning, the Nature Publishing Group (NPG) and the Institute of Electronics, Chinese Academy of Sciences (IECAS) are very proud to launch the co-published academic journal, Microsystems \& Nanoengineering, on May 28, 2015. We consider it as a great honor to write this editorial for the birth of this journal.

NPG is an international publishing company that publishes academic journals, magazines, and online databases in science and medicine. NPG's flagship publication is Nature, a weekly multidisciplinary journal first published in 1869. It also publishes Nature Research journals, Nature Reviews journals, and societyowned academic journals. Microsystems \& Nanoengineering is targeted for one of the important academic journals from NPG.

Established in 1956, the IECAS was the first comprehensive institute in China in the fields of electronics, information science and technology. In China, the Institute of Electronics is recognized as a leader in research in fields including microwave imaging electronics, geospatial information technology, and MEMS transducers. The State Key Laboratory of Transducer Technology (North Base) is one of the top national laboratories in China working on MEMS transducers.

Following the rapid growth of the economy in China over the last 30 years, there is now a strong desire to focus on the development of science and technology for the long-term future. Recognizing the importance of academic journals in disseminating research results and instigating new research ideas, the IECAS will sponsor Microsystems \& Nanoengineering as an international platform, providing the researchers in MEMS and nanotechnology with a unique opportunity to closely interact Chinese researchers. This journal will not only provide a venue for an in-depth scientific exchange, but also seek to promote research collaborations and international funding sources, especially those involving China.

This new joint publication with the prestigious NPG, will provide a rapid forum for the dissemination of original research articles and selected high-quality review articles in all aspects of microsystems and nanoengineering with emphases on fundamental research. We will publish papers alongside more general research summaries to increase readership, create impact, in a manner that will distinguish the new Nature journal from other journals in MEMS and nanoengineering. As editors-in-chief on behalf of the editorial board, the purpose of this editorial is to formally invite you to pay attention to and submit your best work to this new journal, to be distributed to all international laboratories in MEMS \& naonengineering first in 2015. For detailed information about this new journal, please refer to the follow journal website: http://www.nature.com/micronano.

Microsystems \& Nanoengineering is the first engineering journal to be co-published by NPG and IECAS. This journal aims to publish top-quality, peer-reviewed fundamental and applied research in the exciting and emerging fields of microsystems and nanoengineering. Submissions will be welcome in fields including design (theory, modelling, or simulation), fabrication, characterization, reliability, packaging, and applications of devices and systems with micro- or nano-scale features.

Microsystems \& Nanoengineering will be an international peerreviewed journal with a wide-ranging coverage, encompassing research activities in MEMS \& nanotechnology. The journal will cover topics including (1) new physics of micro- and nano-systems, (2) micro- and nano-mechanics, modelling, (3) new materials for micro- and nanosystems, (4) micro- and nano-sensors, (5) micro- and nano-actuators, (6) micro- and nano-fluidics, (7) polymer MEMS and NEMS, (8) biomedical MEMS and NEMS, (9) micro- and nano-optics, optical MEMS (10) energy harvesting and power MEMS, (11) integrated photonics, hybrid optical and electronic integration, (12)nanophotonic systems and circuits, microwave photonics, (13)micro- and nano-engineered systems, integrated microsytems and functional nanosystems, (14) microand nano-fabrication technologies, "top-down" and "bottom-up" technologies, (15) characterization of micro- and nano-systems, and (16) applied sciences of micro- and nano-systems. All aspects of basic and applied research on MEMS/NEMS will be covered in this journal. We will publish original full research papers with more general research summaries and timely state-of-the-art reviews, encompassing the basic and applied research in MEMS/NEMS.

The journal has a distinguished editorial board with extensive academic qualifications, ensuring that the journal maintains high scientific standards and has a broad international coverage in Microsystems \& Nanoengineering. Authors can benefit from the following features from this new journal including (1) open access publication -anyone can download and read your paper, (2) wide exposure to a large global audience on nature.com, (3) internationally renowned editors and editorial board, (4) quality peer-review and fast publication, and (5) research summaries of published papers to interest a broader readership. Please consider to submit your best manuscript on the journal website: http://mts-micronano.nature.com/.

As micro- and nano-technology is increasingly recognized as bringing great benefit to human society, we believe that Microsystems \& Nanoengineering can play a very important role to help in achieving this goal. We want to sincerely thank current and prospective authors, readers, editors, and reviewers for your strong support and future contributions. We wish Microsystems \& Nanoengineering much success for years to come!

\section{COMPETING INTERESTS}

The authors declare no conflict of interest.

Yirong $\mathrm{Wu}$ Editor-in-Chief, Microsystems \& Nanoengineering; Academician and Director, Institute of Electronics, Chinese Academy of Sciences, China

lan White

Executive Editor-in-Chief, Microsystems \& Nanoengineering; Fellow of Royal Academy of Engineering and Professor, University of Cambridge, UK

Tianhong Cui Executive Editor-in-Chief, Microsystems \& Nanoengineering; Professor, University of Minnesota, 111 Church Street S.E., Minneapolis, MN 55455, USA

Tel: 1-612-626-1636 E-mail: tcui@me.umn.edu, tcui@mail.ie.ac.cn

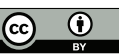

This license allows readers to copy, distribute and transmit the Contribution as long as it is attributed back to the author. Readers are permitted to alter, transform or build upon the Contribution, and to use the article for commercial purposes. Please read the full license for further details at http:// creativecommons.org/licenses/by/4.0 